\title{
Rise of the Phoenix: Mucormycosis in the Context of COVID-19
}

Vanessa Cordeiro Dias*

Federal University of Juiz de Fora, Brazil

\begin{abstract}
Opportunistic infections are especially common in patients who, apart from the current COVID-19 disease, also have other comorbidities such as diabetes, prolonged stay in intensive care unit, treatment with mechanical ventilation, antibiotic therapy and prolonged use of corticosteroids. Mucormycosis, for example, is an acute or sub acute rapidly progressing infections caused by the angioinvasive fungi in the order of Mucorales such as species of Rhizopus, Mucor, Rhizomucor, Syncephalastrum, Cunninghamella, Apophysomyces and Lichtheimia. This infection is characterized by vascular invasion by the fungal hyphae which leads to thrombosis and necrosis. To diagnose the suspicion of mucormycosis, an extensive analysis of the patient's history associated with physical and imaging examination is very important. Biopsy, culture in specific media and polymerase chain reaction (PCR) of clinical specimens can be used to identify the fungus agent of mucormycosis. The widely accepted treatment of choice for mucormycosisis amphotericin B. In addition to early detection of the fungus, the correction of pre-existing pathologies (such as diabetes, immunosuppression and inflammation) is of great importance to ensure therapeutic success. Thus, given this scenario, it is imperative that clinicians should be sensitized to the increased risk of development of this fatal infection, especially while treating diabetic patients with COVID-19, using corticosteroids.
\end{abstract}

Keywords

COVID-19, Mucormycosis, Coinfection, Fungal

\section{Introduction}

At the end of 2019, a new type of coronavirus (SARSCoV-2) appeared in Wuhan/China which, rapidly spreading around the world, has become a huge challenge for the public health. This virus was then named COVID-19 and may vary in severity. The clinical manifestations range from a selflimiting illness with general symptoms, such as fever, cough, headache, to more severe complications, including acute respiratory distress syndrome, sudden cardiac death, liver dysfunction, and multi-organ failure [1,2].

Opportunistic infections are especially common in patients who, apart from the current COVID-19 disease, also have other comorbidities such as diabetes, prolonged stay in intensive care unit, treatment with mechanical ventilation, antibiotic therapy and prolonged use of corticosteroids. These drugs are immunosuppressive and increase blood sugar levels especially in diabetic patients. Both effects contribute to the development of mucormycosis [1-3].

Mucormycosis is an acute or subacute rapidly progressing infections caused by the angioinvasive fungi in the order of Mucorales such as species of Rhizopus, Mucor, Rhizomucor, Syncephalastrum, Cunninghamella, Apophysomyces and Lichtheimia [4]. This infection is characterized by vascular invasion by the fungal hyphae which leads to thrombosis and necrosis. Due to the darkened or black aspect of the lesions, mucormycosis has been called "black fungus" disease. It is important to emphasize that these fungi are not demaceous but hyaline. They are considered saprophytic and can be found commonly in nature, soil, air, food, decaying organic material and even covering the skin or mucosa of healthy individuals [5]. Thus, we can compare phoenix to fungi agents of mucormycosis. There have always been, although underreported as agents of infection in humans, sometimes being considered environmental contaminants. At this moment, when a large contingent of individuals severely ill by COVID-19 is observed, we also observe a new phoenix reappearing. Surgical debridement of the infected area should be performed as soon as possible once the diagnosis is confirmed.

Mucormycosis can present in many forms such as,

*Corresponding author: Vanessa Cordeiro Dias, Federal University of Juiz de Fora, Juiz de Fora, Minas Gerais, Brazil

Accepted: January 29, 2022

Published online: January 31, 2022

Citation: Dias VC (2022) Rise of the Phoenix: Mucormycosis in the Context of COVID-19. Ann Microbiol Res 6(1):140-141 
gastrointestinal (more common in young children), rhino cerebral (more common in people with uncontrolled diabetes and in people who had a transplant), cutaneous, pulmonary (more common in people with cancer and in people who have had a transplant) and disseminated. The most frequent clinical pictures of COVID-19 are rhino cerebral, orbital and pulmonary mucormycosis $[4,5]$.

Patients with COVID-19 ends up becoming an excellent culture medium for the development of fungi, especially those causing mucormycosis, offering ideal conditions, such as ph reduced, raised body temperature (optimal for thermo tolerant organism), raised ferritin and glucose levels (required by fungic metabolism), beyond the endothelial and alveolar damage as port of entry and immunosuppression by drug or cytokine storm, important for the growth of opportunistic organism [6].

To diagnose the suspicion of mucormycosis, an extensive analysis of the patient's history associated with physical and imaging examination is very important. In susceptible patients, a typical finding on a cranial computed tomography will show bone destruction. Cranial nuclear magnetic resonance is recommended for cases where there is brain, sinus and cerebral involvement [7]. Biopsy, culture in specific media and Polymerase Chain Reaction (PCR) of clinical species can be used to identify the fungus agent of mucormycosis [1,7]. The in vitro antifungal susceptibility testing of the fungic isolate should be performed by the micro broth dilution method as the Clinical Laboratory Standards Institute guidelines [8].

Biomarkers such as beta-d-glucan and galactomannan antigens, which aid in the diagnosis of invasive aspergillosis, do not apply to the laboratory diagnosis of mucormycosis [8]. Considering the limited diagnostics available for mucormycosis, an urgent need exists development of noninvasive tests. An enzyme-linked immunospot (ELISpot) assay, which detects mucormycete-specific T-cells, analogous to interferon-gamma-release assays for detection of latent tuberculosis, has been evaluated in haematological patients [9]. Mucorales-specific T-cells cells may be useful in the diagnosis of invasive fungal disease and contribute to control the invasive fungal infection by Mucorales [10].

Surgical debridement of the infected area should be performed as soon as possible once the diagnosis is confirmed. Surgery alone has been reported not to be curative, but an aggressive surgical approach has been shown to improve survival [5].

The widely accepted treatment of choice for mucormycosisis is amphotericin B. When administering amphotericin B, it is essential to monitor kidney function due to its high nephrotoxicity incidence. When extensive disease occurs, second line therapies may be contemplated such as echinocandins and amphotericin B. Some of the other second-line accepted antifungal includes the triazoles, posaconazole and isavuconazole. Patients that are intolerant to amphotericin B are given posaconazoles. Isavuconazole has an extended spectrum. Because of this, it is the only antifungal used in the treatment of invasive mucormycosis [7].
In addition to early detection of the fungus, the correction of pre-existing pathologies (such as diabetes, immunosuppression and inflammation) is of great importance to ensure therapeutic success [3].

Thus, given this scenario, it is imperative that clinicians should besensitized to the increased risk of development of this fatal infection, especially while treating diabetic patients with COVID-19 using corticosteroids. In addition, the rational use of glucocorticoids must be recommended in all patients [3]. Early diagnostic and timely management are necessary to control mucormycosis.

\section{Declaration of Interest Statement}

The author declared no potential conflicts of interest with respect to the research, authorship, and/or publication of this article.

\section{Author's Contribution}

VCD was responsible for conception of idea, literature review and, preparation of manuscript.

\section{Funding}

Not applicable.

\section{References}

1. Szarpak L, Chirico F, Pruc M, et al. (2021) Mucormycosis - A serious threat in the COVID-19 pandemic? J Infect 83: 237-279.

2. Kanwar A, Jordan A, Olewiler S, et al. (2021) A Fatal Case of Rhizopus azygosporus Pneumonia Following COVID-19. J. Fungi 7: 174.

3. Bakshi1 SS, Kalidoss VK (2021) COVID 19 infection and mucormycosis - a dangerously increasing combination. Egypt J Otolaryngol 37: 53.

4. Bhatt K, Agolli A, Patel MH, et al. (2021) High mortality coinfections of COVID-19 patients: Mucormycosis and other fungal infections. Discoveries (Craiova) 9: e126.

5. Sharma S, Grover M, Bhargava S, et al. (2021) Post coronavirus disease mucormycosis: A deadly addition to the pandemic spectrum. J Laryngol Otol 135: 442-447.

6. Pandiar D, Kumar NS, Anand R, et al. (2021) Does COVID 19 generate a milieu for propagation of mucormycosis? Med Hypotheses 15: 110613.

7. Alekseyeva K, Didenkoa L, Chaudhryb B (2021) Rhinocerebral mucormycosis and COVID-19 pneumonia. J Med Cases 12: 85-89.

8. Garg D, Muthu V, Sehgal IS, et al. (2021) Coronavirus disease (Covid-19) associated mucormycosis (CAM): Case report and systematic review of literature. Mycopathologia 2021: 1-10.

9. Potenza L, Vallerini D, Barozzi P, et al. (2011) Mucorales-specific $T$ cells emerge in the course of invasive mucormycosis and may be used as a surrogate diagnostic marker inhigh-risk patients. Blood 118: 5416-5419.

10. Pasero D, Sanna S, Liperi C, et al. (2020) A challenging complication following SARS-CoV-2 infection: A case of pulmonary mucormycosis. Infection 49: 1055-1060. 